\title{
Ex vivo hepatic venography for hepatocellular carcinoma in livers explanted for liver transplantation
}

Kensuke Miyazaki, Akihiko Soyama, Masaaki Hidaka, Koji Hamasaki, Kosho Yamanouchi, Mitsuhisa Takatsuki, Takashi Kanematsu and Susumu Eguchi

\begin{abstract}
Background: Hepatocellular carcinoma (HCC) is supposed to have a venous drainage system to a portal vein, which makes intrahepatic metastasis possible. However, the mechanism of extrahepatic recurrence, including the possibility of a direct route to the systemic circulation from the HCC nodules, remains unclear. Therefore, we performed retrograde hepatic venography for HCC in livers that had been explanted for liver transplantation in order to explore the possible direct connection between the hepatic vein and HCC nodules.

Methods: Of 105 living-donor liver transplantations (LDLT) performed up to July, 2009 at the Department of Surgery, Nagasaki University Hospital, dynamic hepatic venography was performed with contrast media under fluoroscopy for the most recent 13 cases with HCC. The presence of a tumor stain for each HCC case was evaluated and compared with the histological findings of HCC.
\end{abstract}

Results: Hepatic venography revealed a tumor stain in 2 of 13 cases (15\%). Neither showed any microscopic tumor invasion of HCC into the hepatic vein. In the other 11 cases, there were 4 microscopic portal venous invasions and 2 microscopic hepatic venous invasions. No patients have shown HCC recurrence in follow-up (median period, 13 months).

Conclusion: Using ex vivo hepatic venography, a direct connection to the hepatic vein from HCC in whole liver was revealed in 2 cases without demonstrated histopathological invasion to hepatic vein for the first time in the literature. The finding suggests that there is direct spillage of HCC cells into the systemic circulation via hepatic vein.

\section{Introduction}

Hepatocellular carcinoma (HCC) is one of the most common malignant tumors and the third most common cause of cancer-related death in the world [1]. Despite recent advances in treatments of $\mathrm{HCC}$, the long-term survival of patients with $\mathrm{HCC}$ is still unsatisfactory [2]. Intrahepatic or extrahepatic recurrence usually develops, even after a curative liver resection or a total hepatectomy for orthotopic liver transplantation. There are two mechanisms that are well known for intrahepatic recurrence of HCC: multicentric carcinogenesis due to the underlying liver disease and intrahepatic metastasis with

\footnotetext{
* Correspondence: sueguchi@nagasaki-u.ac.jp
Department of Surgery, Nagasaki University Graduate School of Biomedical

* Correspondence: sueguchi@nagasaki-u.ac.jp
Department of Surgery, Nagasaki University Graduate School of Biomedical Sciences, Nagasaki, Japan
}

() 2011 Miyazaki et al; licensee BioMed Central Ltd. This is an Open Access article distributed under the terms of the Creative Commons Attribution License (http://creativecommons.org/licenses/by/2.0), which permits unrestricted use, distribution, and reproduction in any medium, provided the original work is properly cited. venous drainage to a portal vein [3]. On the other hand, the mechanism of extrahepatic recurrence of HCC is still controversial, with the possibility of a direct route from HCC nodules to the systemic circulation still unconfirmed. According to previous reports, the predictors for extrahepatic recurrence of HCC are the size and number of tumors, vascular invasion, and elevated tumor markers [4-8]. However, even cases with small and single HCC lesions sometimes develop extrahepatic recurrence.

In this study, we performed hepatic venography for $\mathrm{HCC}$ in explanted livers for liver transplantation, in order to explore the direct connection between the hepatic vein and HCC nodules. 


\section{Methods \\ Patients}

One hundred five living-donor liver transplantations (LDLT) were performed up to July, 2009 at the Department of Surgery, Nagasaki University Hospital. Of these 105 LDLTs, we performed hepatic venography on explanted livers of the most recent 13 cases, which detected HCC lesions preoperatively and/or postoperatively. There were 9 males and 4 females with a median age at LDLT of 59 years (range; 52-68 years) (Additional file 1, Table S1).

\section{Ex vivo hepatic venography}

Using livers explanted for LDLT, hepatic venography was performed with contrast media (Urografin, Nihon Schering, Osaka, Japan) under fluoroscopy. First, a purse-string suture was placed around the orifice of the right hepatic vein and the hepatic venous trunk of the middle and left hepatic vein, respectively, to prevent the backflow of the contrast media. Second, a plastic needle was cannulated into the hepatic vein and tightened up with the purse-string suture. Third, the contrast media was injected into the hepatic vein by a slow retrograde bolus injection with very low pressure manually (Figure 1). Thereafter, $X$-ray images were taken from several different angles in a series.

\section{Evaluation of hepatic venography}

We defined the venographic positve case as the presence of a tumor stain corresponding to an HCC nodule contiguous with hepatic vein, and smoothly filled with contrast media without any resistance. The presence of a

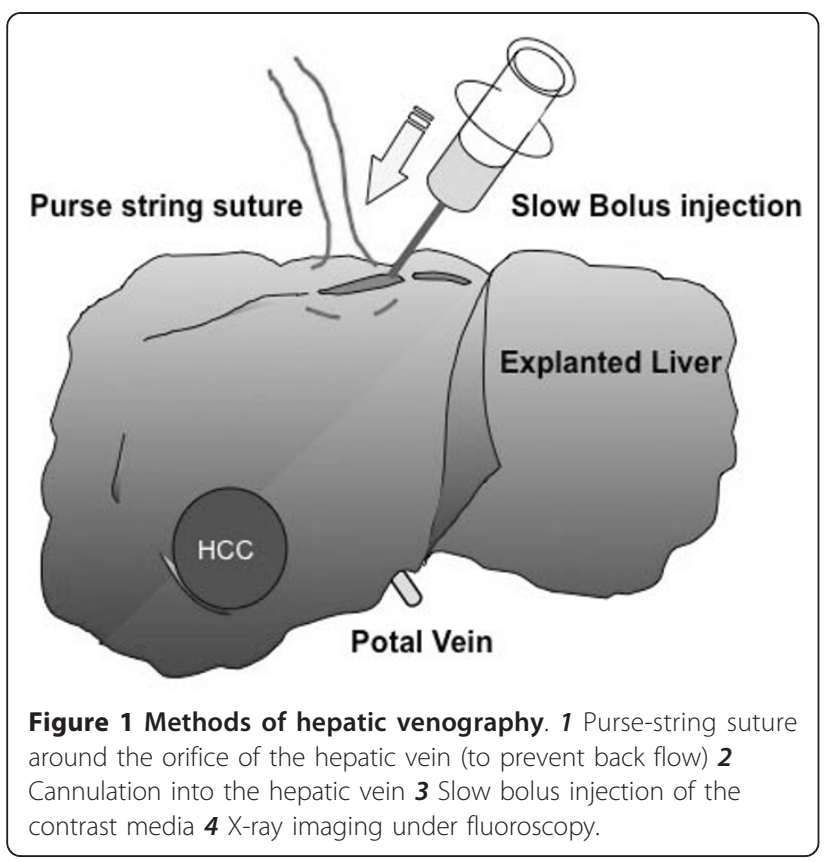

tumor stain corresponding to an HCC nodule was judged to be "positive", while the absence of a tumor stain was judged to be "negative" on the serial $\mathrm{x}$-ray images. The judgment was made by two or three surgeons.

\section{Statistical analysis}

Fisher's exact test was used for the data analysis. A level of $P<0.05$ was considered to indicate statistical significance.

\section{Results}

\section{Ex vivo hepatic venography}

In 2 of 13 cases (15\%), tumor stains were confirmed in a corresponding lesion to indicate the location of an HCC nodule. One positive case was a 65 -year-old male with 2.2-cm HCC located at segment six, associated with hepatitis $\mathrm{C}$ virus-related cirrhosis. The tumor stain from the right hepatic vein was clearly detected with contrast media by retrograde hepatic venography (Figure 2). The other positive case was a 68-year-old male having a 3.2$\mathrm{cm}$ HCC at segment six. A tumor stain was also seen by retrograde hepatic venography from the right hepatic vein (image not shown). The other 11 cases were judged negative by hepatic venography (Additional file 2, Table S2). Though negative cases showed no tumor stains with contrast media, they did show venous compression from the tumor (Figure 3).
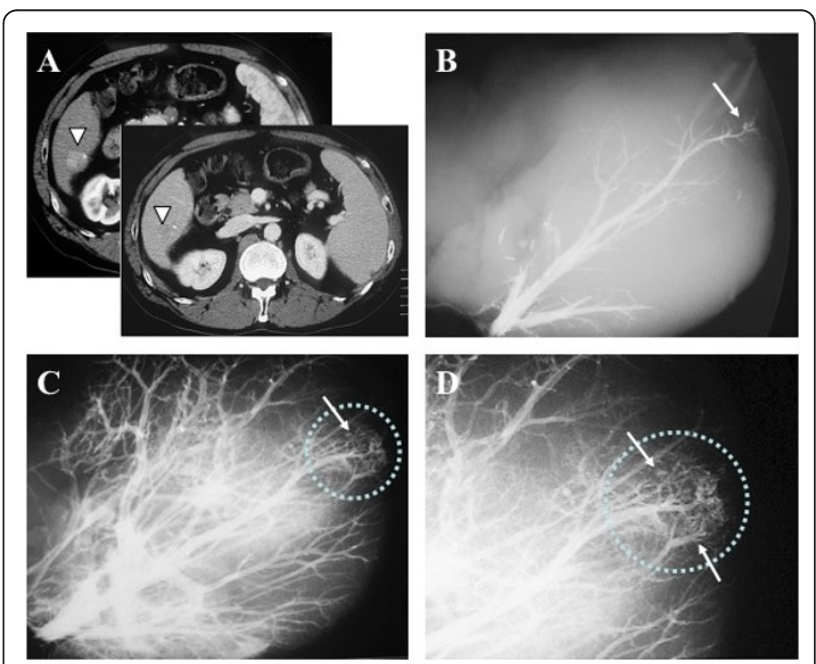

Figure 2 A hepatic venography-positive case. A 65-year-old male with a 2.2-cm HCC at segment 6 (white arrowheads on CT). The broken circle indicates the site of the tumor. Hepatic venography shows tumor stains (arrows) corresponding with an HCC nodule. A: Enhanced CT B: Early phase image of hepatic venography $\mathbf{C}$ : Late phase image of hepatic venography $\mathbf{D}$ : magnified image of the late phase. 

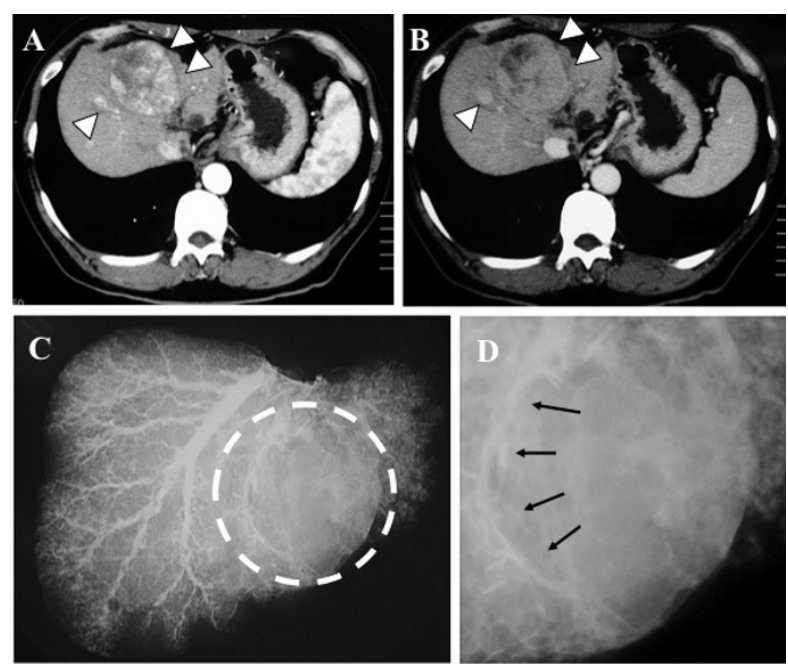

Figure 3 A hepatic venography-negative case. A 58-year-old male with multiple HCCs. A 6.2-cm HCC at segment 4 (white arrowheads). The broken circle indicates the site of the tumor. Hepatic venography shows no tumor stain but venous compression from the tumor (arrows). A: Enhanced CT, early phase B: Enhanced $\mathbf{C T}$, delayed phase $\mathbf{C}$ : Image of hepatic venography $\mathbf{D}$ : Magnified image of hepatic venography.

The relationship between hepatic venography and histopathological findings

Histopathologically, there were 4 portal venous invasions and 2 hepatic venous invasions among the 13 cases. Of the 2 positive hepatic venography cases, one showed portal venous invasion, but neither showed microscopic hepatic venous invasion. No statistically significant relationship was found between tumor stains by ex vivo hepatic venography and microscopic hepatic venous invasion in positive hepatic venography cases.

\section{Outcomes after LDLT}

Five of the patients who underwent hepatic venography died. Two patients died of sepsis, and the other three died due to liver failure. However, no recurrent HCC was found in the follow-up (median period, 25 months) of the 13 cases, including the two patients with venographically demonstrable HCC, who had follow-up periods of 35 and 25 months after LT, respectively, at this writing (Additional file 2, Table S2).

\section{Discussion}

In this study, we have demonstrated a direct connection between primary HCC nodules and the systemic circulation (vena cava) by retrograde hepatic venography with livers explanted for liver transplantation. To our knowledge, this is the first report to visualize the direct communication from HCC nodules to the systemic circulation in explanted whole livers from liver transplantation. In this study, 2 of 13 cases showed tumor stains, which indicate direct venous drainage to the hepatic vein (or vena cava), by hepatic venography. The stains might represent $\mathrm{HCC}$ cells or tumor thrombi spilled from the primary HCC into systemic circulation and thereby likely to be carried to distant organs by the bloodstream. In spite of the presence of a direct connection to the hepatic veins, neither of the two positive cases showed microscopic hepatic venous invasion. These results suggest that the route of the cancer cells into the vessels could be independent of histopathological invasion.

Based on previous reports, various factors are thought to contribute to extrahepatic recurrence; for instance, Funaki et al. reported hematogenous spreading of HCC cells from the primary tumor [9]. Recently, some studies have reported that adhesion molecules, such as E-cadherin $[10,11]$ or CD44 $[12,13]$, play an important role in the extrahepatic recurrence of HCC after hepatectomy or liver transplantation. Other reports have indicated that the presence of cancer stem cells is a key factor $[14,15]$. In any case, cancer cells from the primary lesion likely migrate into the bloodstream of systemic circulation to form metastatic foci in distant organs.

Moreover, several factors seem to be involved in the occurrence of distant metastasis, such as 1) escape from local immunity, 2) connection to systemic circulation, 3) spilling of HCC cells from the primary lesion into the bloodstream, 4) escape from the host immune surveillance systems, 5) adhesion to another organ, and 6) growth. A recent study represents a case of metastasis without pathological venous invasion. Sugino et al. $[16,17]$ described sinusoidal angiogenesis as a non-invasive mechanism of blood-borne metastasis in HCC; i.e., an invasion-independent metastasis pathway. This may suggest that patients after liver transplantation need particularly vigilant observation for extrahepatic recurrence because of their immunosuppressive states.

In addition, the possibility of intrahepatic metastasis as well as extrahepatic metastasis via systemic circulation has also been reported [18]. Thus, patients having HCC with a direct connection to systemic circulation should be monitored, not only for extrahepatic recurrence but also for intrahepatic recurrence, even after liver transplantation.

There are some reports of efferent vessels of HCC. Mitsunobu et al. [19] demonstrated that the portal vein serves as an efferent vessel in advanced HCC by direct injection of radiopaque media into $\mathrm{HCC}$ nodules of resected specimens. Other reports have made similar conclusions from different points of view, namely, histopathological study [20] or color Doppler imaging examination using ultrasonography [21]. Those previous reports suggested the following mechanism. The efferent vessel of hepatic tumors is basically the hepatic vein; 
blood from the HCC still flows out to the hepatic vein at its early stage. With the progress of $\mathrm{HCC}$, the portal vein also acts as an efferent vessel. It is supposed that a capsule is formed as the HCC undergoes dedifferentiation, resulting in regurgitation of blood to the portal vein with the rising internal pressure of HCC nodule. This causes intrahepatic metastasis through the portal vein as well.

In regard to the outcomes of LDLTs for the 13 patients, there has been no recurrence of HCC so far. This may be due to the fact that the follow-up periods are not very long ( 37 months at most), and that all cases except one were within the Milan criteria [22].

\section{Conclusion}

Hepatic venography of 2 of 13 livers explanted for HCC-related LDLT revealed a direct connection between primary HCC nodules and the hepatic vein. Such cases should be strictly observed for extrahepatic and intrahepatic recurrence, even in cases within the Milan criteria and without microscopic hepatic venous invasion.

\section{Additional material}

Additional file 1: Table S1. Patients Characteristics.

Additional file 2: Table S2. Tumor characteristics and clinical outcomes of patients who underwent hepatic venography.

\section{Authors' contributions}

SE designed and coordinated the study. KM, AS, MH, KH performed and carried out the hepatic venography, and KM wrote the manuscript. All authors evaluated the results of the hepatic venography. KY, MT, TK, SE supervised in critically reviewed the manuscript. All authors contributed significantly to this work, and approved the final manuscript.

\section{Competing interests}

The authors declare that they have no competing interests.

Received: 5 April 2011 Accepted: 27 September 2011

Published: 27 September 2011

\section{References}

1. Botha JF, Langnas AN: Liver transplantation for hepatocellular carcinoma: an update. J Natl Compr Canc Netw 2006, 8:762-767.

2. Eguchi S, Kanematsu T: Liver Cancer Study Group of Japan: Comparison of the outcomes between an anatomical subsegmentectomy and a nonanatomical minor hepatectomy for single hepatocellular carcinomas based on a Japanese nationwide survey. Surgery 2008, 143:469-475.

3. Mitsunobu M, Toyosaka A, Oriyama T, Okamoto E, Nakao N: Intrahepatic metastases in hepatocellular carcinoma: the role of the portal vein as an efferent vessel. Clin Exp Metastasis 1996, 14:520-529.

4. Okuda K, Ohtsuki T, Obata H, Tomimatsu M, Okazaki N, Hasegawa H, Nakajima Y, Ohnishi K: Natural history of hepatocellular carcinoma and prognosis in relation to treatment Study of 850 patients. Cancer 1985, 56:918-928.

5. The Cancer of the Liver Italian Program (CLIP) Investigators: A new prognostic system for hepatocellular carcinoma: a retrospective study of 435 patients. Hepatology 1998, 28:751-755.
6. Llovet JM, Bru C, Bruix J: Prognosis of hepatocellular carcinoma: the BCLC staging classification. Semin Liver Dis 1999, 19:329-338.

7. Kanda M, Tateishi R, Yoshida H, Sato T, Masuzaki R, Ohki T, Imamura J, Goto T, Yoshida H, Hamamura K, Obi S, Kanai F, Shiina S, Omata M: Extrahepatic metastasis of hepatocellular carcinoma: incidence and risk factors. Liver Int 2008, 28:1256-1263.

8. Yang Y, Nagano H, Ota H, Morimoto O, Nakamura M, Wada H, Noda T, Damdinsuren B, Marubashi S, Miyamoto A, Takeda Y, Dono K, Umeshita K, Nakamori S, Wakasa K, Sakon M, Monden M: Patterns and clinicopathologic features of extrahepatic recurrence of hepatocellular carcinoma after curative resection. Surgery 2007, 141:196-202.

9. Funaki NO, Tanaka J, Seto SI, Kasamatsu T, Kaido T, Imamura M: Hematogenous spreading of hepatocellular carcinoma cells: possible participation in recurrence in the liver. Hepatology 1997, 25:564-568.

10. Soyama A, Eguchi S, Takatsuki M, Kawashita Y, Hidaka M, Tokai H, Nagayoshi S, Mochizuki S, Matsumoto S, Hamasaki K, Tajima Y, Kanematsu T: Significance of the serum level of soluble E-cadherin in patients with HCC. Hepatogastroenterology 2008, 55:1390-1393.

11. Huang GT, Lee HS, Chen CH, Sheu JC, Chiou LL, Chen DS: Correlation of Ecadherin expression and recurrence of hepatocellular carcinoma. Hepatogastroenterology 1999, 46:1923-1927.

12. Hirohashi K, Yamamoto T, Uenishi T, Ogawa M, Sakabe K, Takemura S, Shuto T, Tanaka H, Kubo S, Kinoshita H: CD44 and VEGF expression in extrahepatic metastasis of human hepatocellular carcinoma. Hepatogastroenterology 2004, 51:1121-1123.

13. Endo K, Terada T: Protein expression of CD44 (standard and variant isoforms) in hepatocellular carcinoma: relationships with tumor grade, clinicopathologic parameters, p53 expression, and patient survival. $J$ Hepatol 2000, 32:78-84.

14. Kamohara Y, Haraguchi N, Mimori K, Tanaka F, Inoue H, Mori M, Kanematsu T: The search for cancer stem cells in hepatocellular carcinoma. Surgery 2008, 144:119-124.

15. Yang ZF, Ngai P, Ho DW, Yu WC, Ng MN, Lau CK, Li ML, Tam KH, Lam CT, Poon RT, Fan ST: Identification of local and circulating cancer stem cells in human liver cancer. Hepatology 2008, 47:919-928.

16. Sugino T, Yamaguchi T, Ogura G, Saito A, Hashimoto T, Hoshi N, Yoshida S, Goodison S, Suzuki T: Morphological evidence for an invasionindependent metastasis pathway exists in multiple human cancers. BMC Med 2004, 5:2-9.

17. Sugino T, Yamaguchi T, Hoshi N, Kusakabe T, Ogura G, Goodison S, Suzuki T: Sinusoidal tumor angiogenesis is a key component in hepatocellular carcinoma metastasis. Clin Exp Metastasis 2008, 25:835-841.

18. Sakon M, Nagano H, Nakamori S, Dono K, Umeshita K, Murakami T, Nakamura $\mathrm{H}$, Monden M: Intrahepatic recurrences of hepatocellular carcinoma after hepatectomy: analysis based on tumor hemodynamics. Arch Surg 2002, 137:94-99.

19. Toyosaka A, Okamoto E, Mitsunobu M, Oriyama T, Nakao N, Miura K. Pathologic and radiographic studies of intrahepatic metastasis in hepatocellular carcinoma; the role of efferent vessels. HPB Surg 1996, 10:97-103

20. Nakashima Y, Nakashima O, Hsia CC, Kojiro M, Tabor E: Vascularization of small hepatocellular carcinomas: correlation with differentiation. Liver 1999, 19:12-18.

21. Tochio H, Kudo M: Afferent and efferent vessels of premalignant and overt hepatocellular carcinoma: observation by color Doppler imaging. Intervirology 2004, 47:144-153.

22. Mazzaferro V, Regalia E, Doci R, Andreola S, Pulvirenti A, Bozzetti F, Montalto F, Ammatuna M, Morabito A, Gennari L: Liver transplantation for the treatment of small hepatocellular carcinomas in patients with cirrhosis. N Engl J Med 1996, 14;334:693-699.

doi:10.1186/1477-7819-9-111

Cite this article as: Miyazaki et al:: Ex vivo hepatic venography for hepatocellular carcinoma in livers explanted for liver transplantation. World Journal of Surgical Oncology 2011 9:111. 